\title{
Potential Nematicidial Activity of Silver Nanoparticles Against the Root-Knot Nematode (Meloidogyne Incognita)
}

\author{
Gregory C. Bernard ${ }^{1 *}$, Jacob Fitch ${ }^{1}$, Byungjin Min ${ }^{2}$, Naresh Shahi' ${ }^{2}$, Marceline Egnin ${ }^{1}$, Inocent Ritte ${ }^{1}$, Willard \\ E Collier ${ }^{3}$, and Conrad Bonsi ${ }^{1}$ \\ ${ }^{1}$ Department of Agriculture \& Environmental Sciences, USA
}

${ }^{2}$ Department of Food and Nutritional Sciences, Tuskegee University, USA

${ }^{3}$ Department of Chemistry, Tuskegee University, USA

*Corresponding author: Gregory C Bernard, Department of Agriculture \& Environmental Sciences, USA

Received Date: August 16, 2018

Published Date: September 11, 2019

\begin{abstract}
Plant-parasitic nematodes (PPNs) are incredibly damaging pests, which cause significant losses in crop yields worldwide. One of the most prevalent PPNs is the root-knot nematode (Meloidogyne spp.) ranks number one on the most economically devastating list of pests and thus scientifically important PPNs. Recently, the use of chemical nematicides for root-knot nematode management has decreased due to governmental restrictions; which necessitates the development and identification of alternative pest management procedures. In this study, we evaluated the use of silver nanoparticles (AgNPs) as a potential biopesticide under in-vitro conditions. AgNPs were synthesized utilizing a naturally occurring biopolymer (chitosan) as a reducing agent through microwave irradiation. When J2-stage nematodes were exposed to $0.0005 \mu \mathrm{g}$ of AgNPs for $1 \mathrm{~min}$, significant mortality ( $\mathrm{P} \leq 0.01)$ was observed and approximately $100 \%$ of nematodes became inactive within 24 and 48 hrs. Our preliminary study has demonstrated a potential environmentally friendly alternative for the management of the root-knot nematodes.
\end{abstract}

Keywords: Root-knot nematode, Silver nanoparticles, Biopesticide, Disease, Plants

\section{Introduction}

Each year, plant-parasitic nematodes (PPNs) cause over $\$ 100$ billion in damages to crops [1]. The most scientifically and economically essential PPNS include cyst nematodes (Heterodera and Globodera spp.) root-lesion nematodes (Pratylenchus spp.); the burrowing nematode Radopholus similes; Ditylenchus dipsaci, and root-knot nematodes (Meloidogyne spp.) which ranks number one [2]. The use of chemical pesticides typically maintains nematode control; however, the overuse of such chemical agents has resulted in a negative environmental impact Sande D, et al. [3] thus, alternative methods of nematode control which are environmentally friendly and cost-effective are of great interest to researchers and plant breeders. Silver nanoparticles (AgNPs) are a well-known example of nano-sized materials that have been applied as a means of controlling human pathogenic microbes. AgNPs possess antimicrobial effects and have been frequently used in the field of medicine [4]. Recently, nanotechnology has been successfully applied to pest management in crops Chhipa $\mathrm{H}$ [5] and the use of silver nanoparticles (AgNPs) has been shown to demonstrate anti-nematode effects Cromwell WA, et al. [6] Silver nanoparticles are often synthesized employing harsh chemicals including sodium borohydride (NaBH4) as a primary reductant [7]. In this objective, we seek to develop AgNP pesticides from reducing agents which are natural biological polymers to potentially mitigate damages caused by root-knot nematodes. Overall, the development of such biopesticides may provide an alternative means of nematode control that are environmentally friendly and as efficacious as the conventional high-risk synthetic pesticides commonly used.

\section{Materials and Methods}

\section{Silver nanoparticle synthesis:}

Method: AgNPs were synthesized by microwave technique Seku K, et al. [8] using low molecular weight chitosan as a reducing agent. Chitosan is derived from chitin, a naturally occurring polymer of $\mathrm{N}$-acetylglucosamine, found in the exoskeleton of crustaceans and in the cell wall of fungi. Field Emission Scanning Electron Microscopy (FE-SEM) images analysis showed that the 
size and shape of AgNPs in nanoscale and spherical, which were homogeneously dispersed into the biopolymer matrix (chitosan) (Figures 1\&2). Chitosan-derived AgNPs were homogenized in $10 \mathrm{ml} 1 \%$ Lactic acid for a final working solution of $1 \mathrm{~mL}$ of $1 \%$ lactic acid containing 0.1 ug of AgNPs and 0.01gram of chitosan Chitosan-derived AgNPs were solubilized in $10 \mathrm{ml} 1 \%$ Lactic acid for treatment.

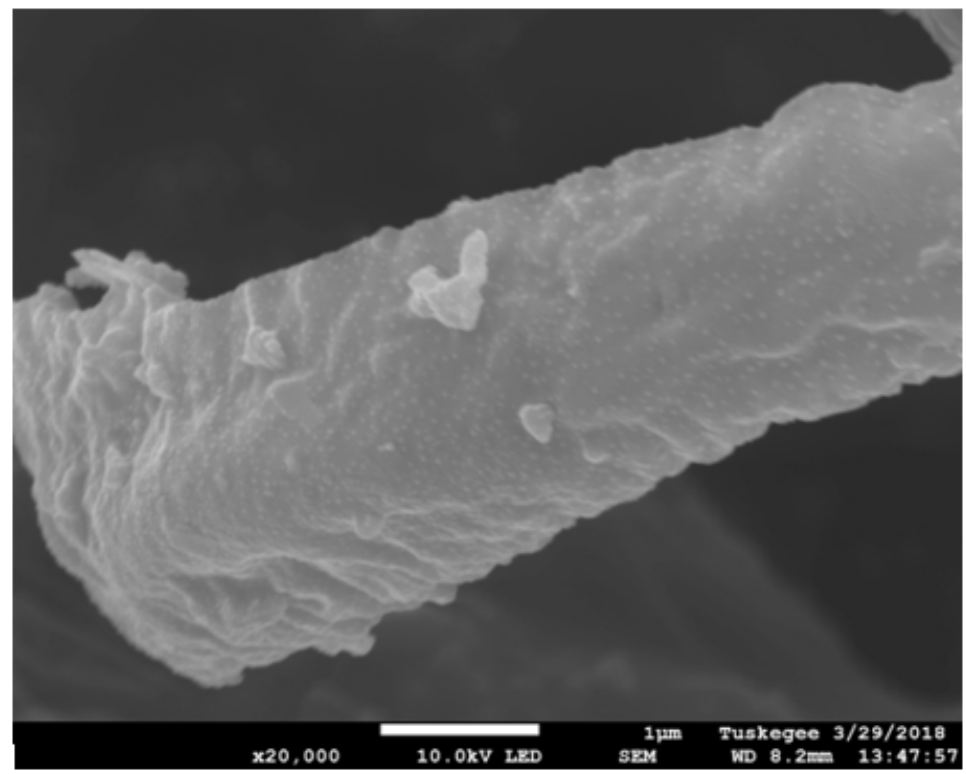

Figure 1: FE-SEM images of homogeneously dispersed AgNPs into Chitosan matrix at 20, $000 \mathrm{X}$.

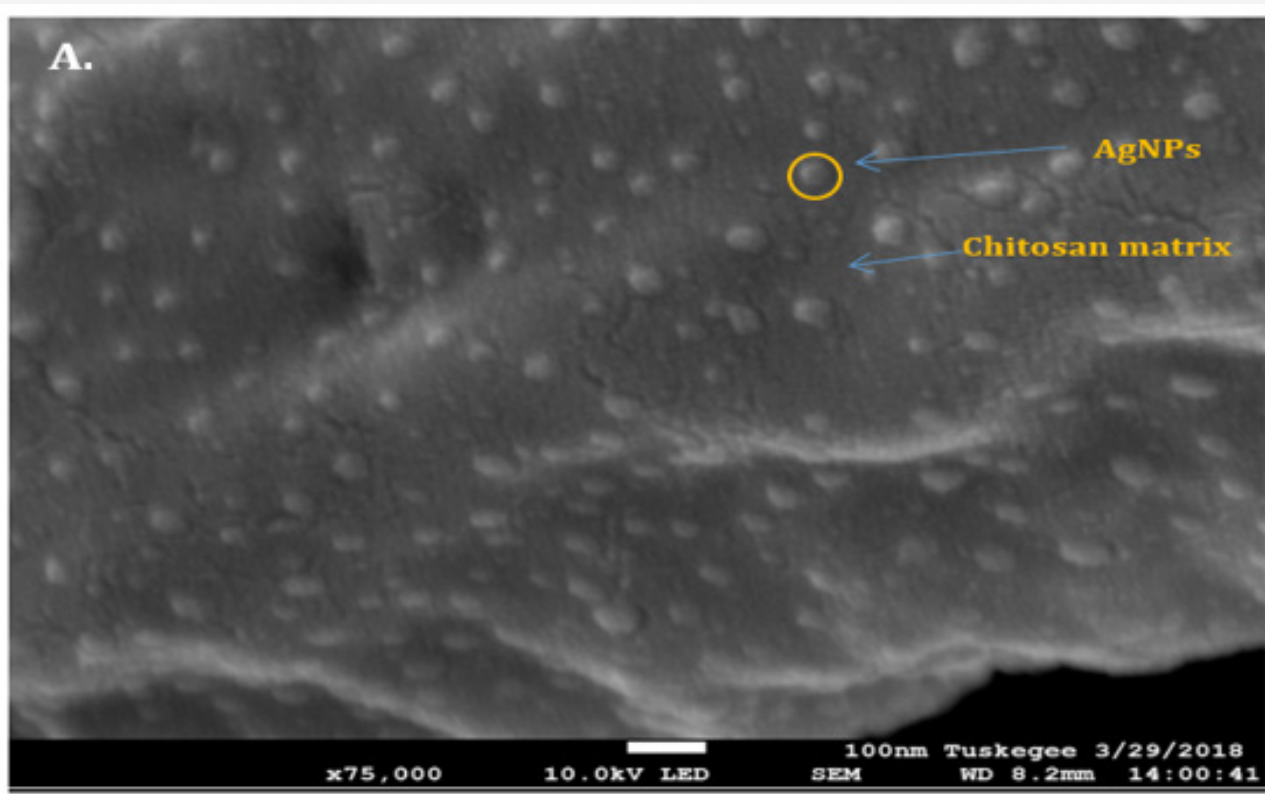

Figure 2: FE-SEM images of homogeneously dispersed AgNPs into Chitosan matrix at $75,000 \mathrm{X}$

\section{Nematode collection and preparation}

Meloidogyne incognita race three juveniles were collected from infested soil and suspended in $1 \mathrm{ml}$ of sterile tap water. Approximately 100-160 nematodes were placed in $1 \mathrm{ml}$ microcentrifuge tubes and treated with 0.0005 ug AgNPs suspended in $0.05 \mathrm{mg}$ chitosan/0.005\% lactic acid at $1 \mathrm{~min}, 24 \mathrm{hr}$ and $48 \mathrm{hr}$ intervals, incubated at $28^{\circ} \mathrm{C}$. Nematodes treated with $0.05 \mathrm{mg}$ ug of chitosan and $0.005 \%$ lactic acid served as controls. The total number of living and dead nematodes from treated and control groups were quantitated using a gridded nematode counting dish and each time interval replicated three independent times. Dead nematodes were confirmed by fine needle probing [1]. Due to the dramatic difference in nematode numbers between treated control groups, an ANOVA Single Factor analysis ( $\mathrm{P} \leq 0.05)$ was used to identify significant differences in the mean number of living and dead nematodes between groups.

\section{Results}

After AgNPs treatment, dead nematodes exhibited the typical "banana shape" curvature predominately displayed in treated groups (Figures 3,4). At the 1-min exposure interval, a significant difference $(\mathrm{P}<0.01)$ in living and dead nematodes between treated and control groups was observed(Figure 5). The 24 and $48 \mathrm{hr}$ AgNPs exposure groups showed a $100 \%$ mortality in comparison to the controls (Figures 6,7). 


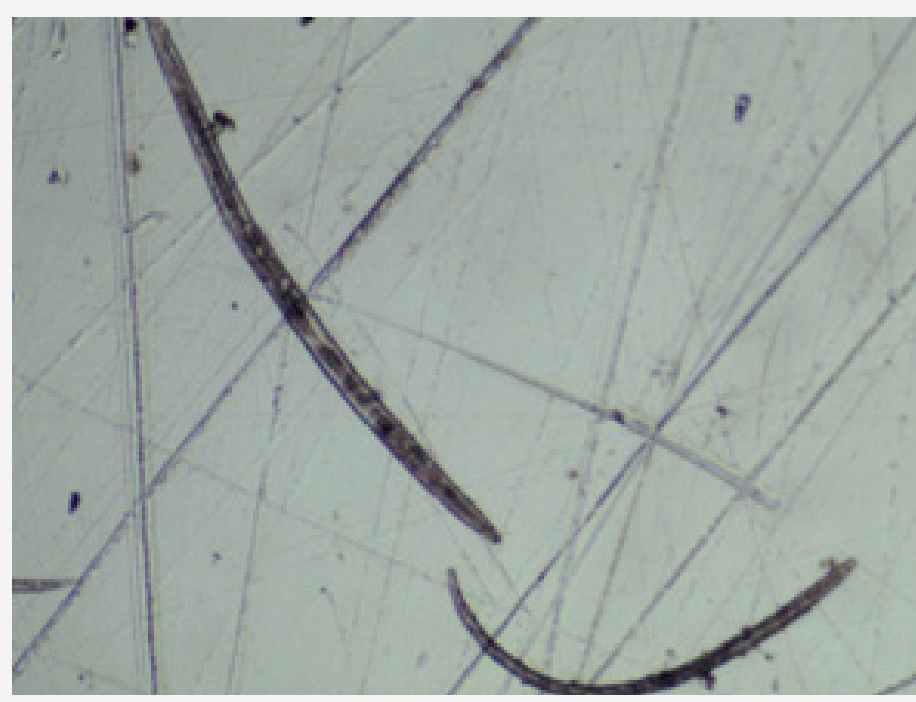

Figure 3: Second-stage M. incognita root-knot nematodes from AgNPs exposed groups..

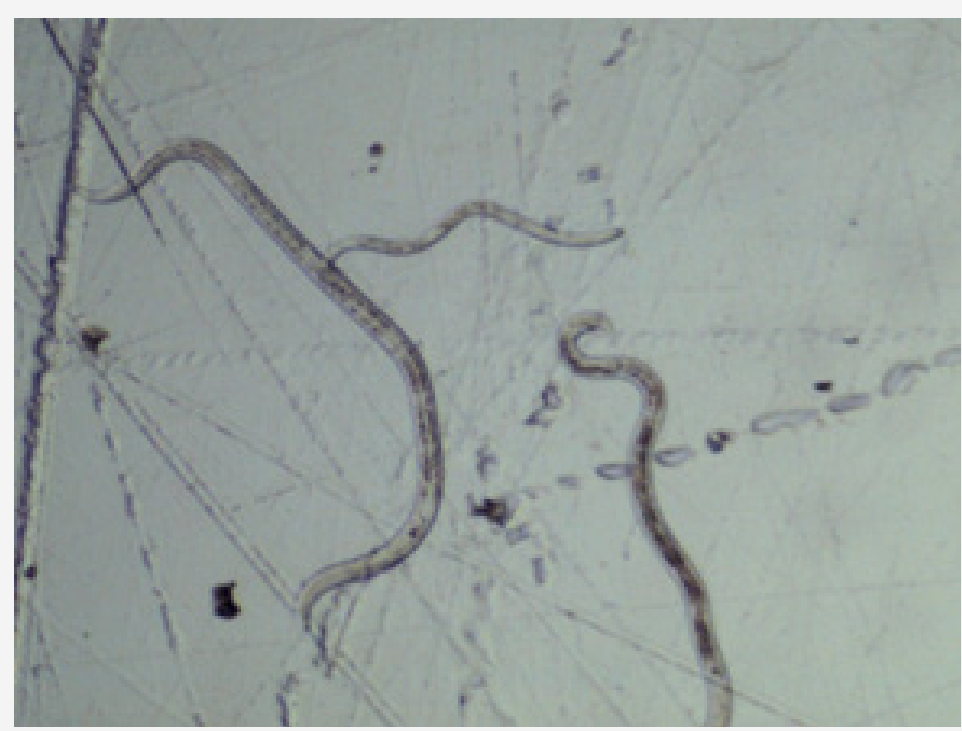

Figure 4: Second-stage M. incognita root-knot nematodes from control groups.

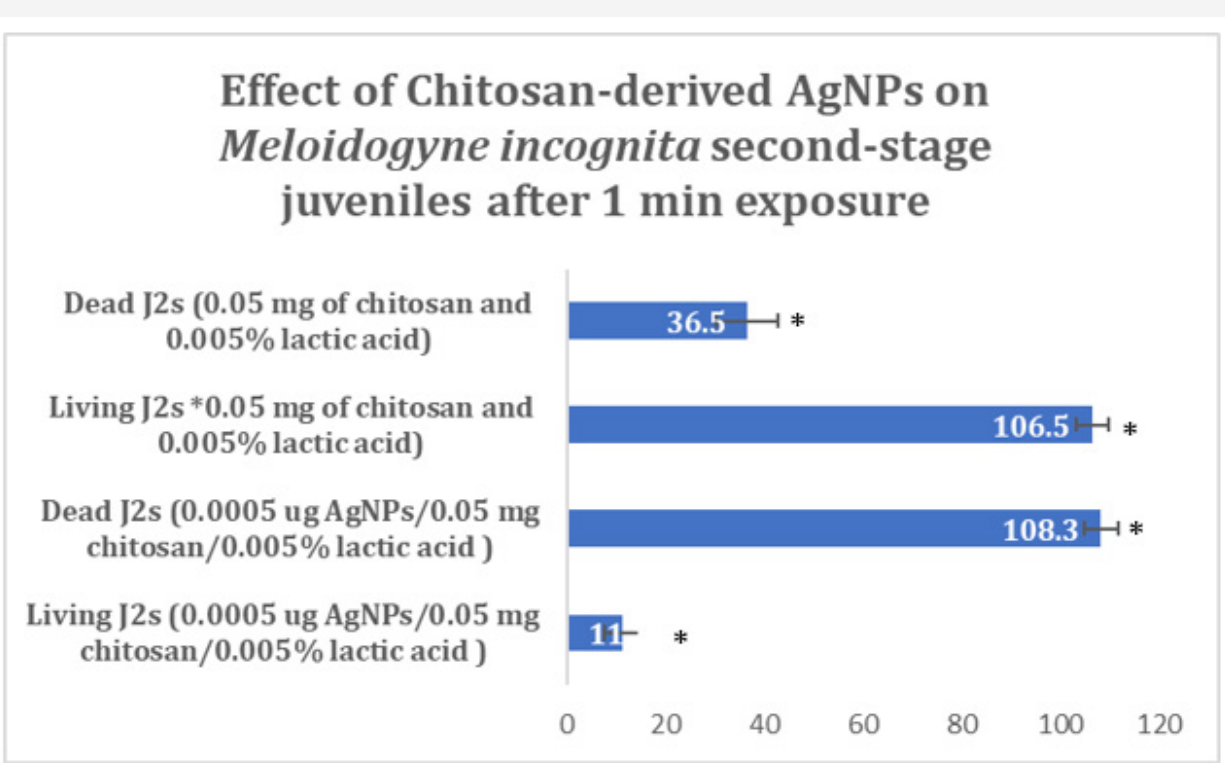

Figure 5: Effect of Chitosan-derived AgNPs on Meloidogyne incognita second stage juveniles after 1 min exposure. 


\section{Effect of Chitosan-derived AgNPs on \\ Meloidogyne incognita second-stage juveniles after $24 \mathrm{hr}$ exposure}

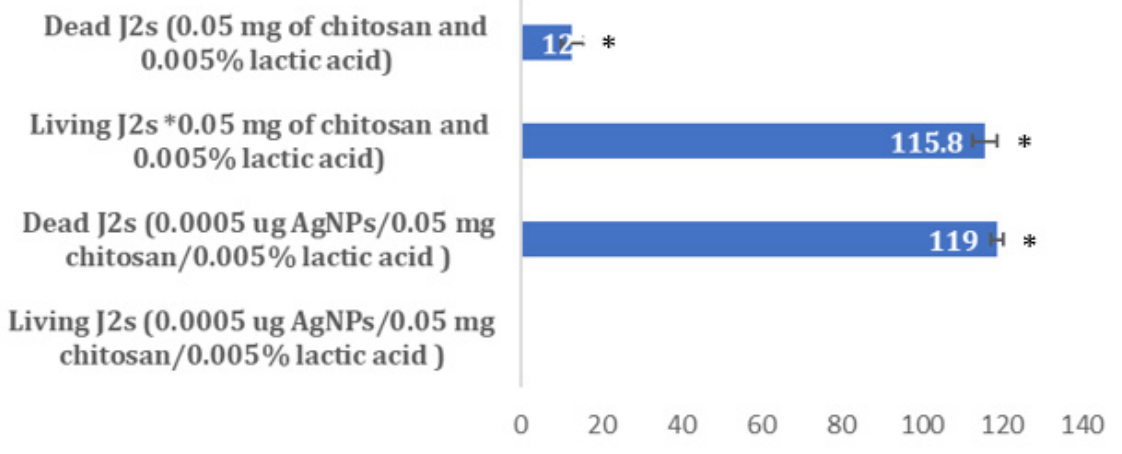

Figure 6: Effect of Chitosan-derived AgNPs on Meloidogyne incognita second stage juveniles after $24 \mathrm{hr}$ exposure.

\section{Effect of Chitosan-derived AgNPs on Meloidogyne incognita second-stage juveniles after $48 \mathrm{hr}$ exposure}

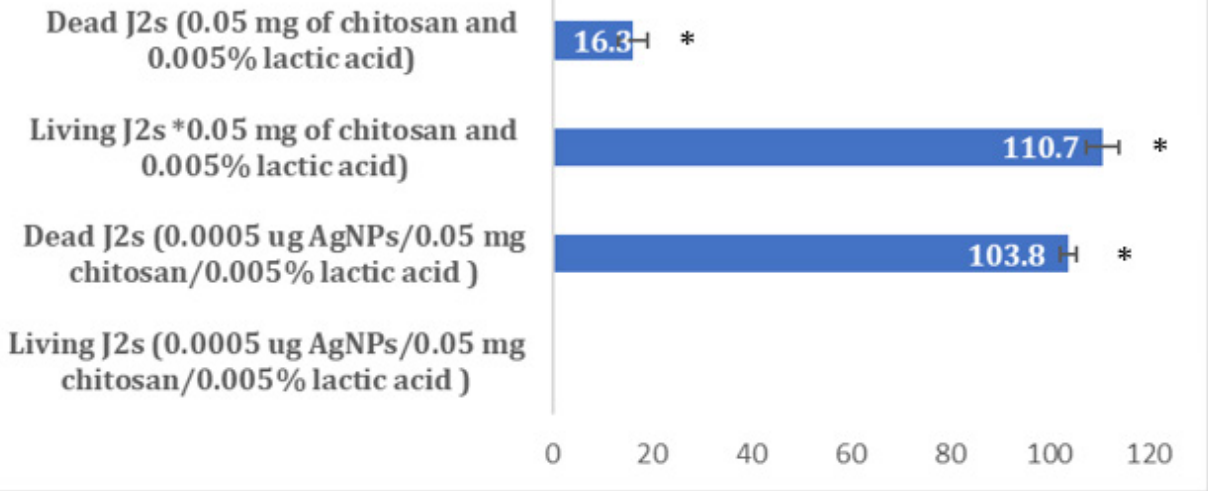

Figure 7: Effect of Chitosan-derived AgNPs on Meloidogyne incognita second stage juveniles after 48 hr exposure.

\section{Conclusion}

Our study has shown a potential nematicidial effect of Chitosanderived AgNPs on root-knot nematodes. Interestingly, different molecular weights of chitosan has shown nematicidal activity on root-knot nematodes [10]. Although the antimicrobial mechanism of AgNPs on microorganisms has been poorly elucidated, the harmful impact of AgNPs may be correlated to the development of free radicals from the silver surface leading to an increase in oxidative stress and membrane damage [11]. Interestingly, in response to the pathogen invasion, plants regulate the expression of free radicals during defense signaling pathway activation [12]. Future experiments will include concentration optimizations of AgNPs and its reducing agent potentials prior to applying to greenhouse and field trials for efficacy determination on nematode burdens during plant cultivation [13].

\section{Acknowledgment}

Supported byUSDAUSDA-NIFA1890Grant\#2014-38821-22448; Tuskegee University George Washington Carver Agricultural Experiment Station (GWCAES), and USDA NIFA-EVANS-ALLEN.

\section{Conflict of interest}

No conflict of interest.

\section{References}

1. Abad P, Gouzy J, Aury J, Castagnone Sereno P, Danchin E, et al. (2008) Genome sequence of the metazoan plant-parasitic nematode Meloidogyne incognita. Nat Biotechnol 26(8): 909-15.

2. Jones J, Haegeman A, Danchin E, Gaur H, Helder J, et al. (2013) Top 10 plant-parasitic nematodes in molecular plant pathology. Mol Plant Pathol 14(9): 946-961.

3. Sande D, Mulle J, Wetzstein M, Houston J (2011) Environmental Impacts from Pesticide Use: A Case Study of Soil Fumigation in Florida Tomato Production. Int J Environ Res Public Health 8(12): 4649-4661. 
4. Xiang Y, Li J, Liu X, Cui Z, Yang X et al. (2017) Construction of poly (lactic co-glycolic acid)/ZnO nanorods/Ag nanoparticles hybrid coating on $\mathrm{Ti}$ implants for enhanced antibacterial activity and biocompatibility. Mater Sci Eng C Mater Biol Appl 79: 629-637.

5. Chhipa H (2017) Nanofertilizers and nanopesticides for agriculture. Environmental Chemistry Letters 15(1): 15-22.

6. Cromwell WA, Yang J, Starr JL, Jo YK (2014) Nematicidal Effects of Silver Nanoparticles on Root-knot Nematode in Bermudagrass. J Nematol 46(3): 261-266.

7. Bernard Gregory C, Egnin Marceline, Bonsi Conrad, Mortley Desmond, Witola H William, et al. (2017) Evaluation of root-knot nematode resistance in sweetpotato. African Journal of Agricultural Research. 12(16): 1411-1414.

8. Agnihotri S, Mukherji, Mukherji S (2014) Size-controlled silver nanoparticles synthesized over the range 5-100 nm using the same protocol and their antibacterial efficacy. RSC Advances 4(8): 3974-3983.
9. Seku K, Gangapuram BR, Pejjai B (2018) Microwave-assisted synthesis of silver nanoparticles and their application in catalytic, antibacterial and antioxidant activities. J Nanostruct Chem 8: 179.

10. Abbasi WM, Ahmed N, Zaki JM, Shaukat SS (2008) Effect of Barleria acanthoides Vahl. on root-knot nematode infection and growth of infected okra and brinjal plants. Pak J Bot 40(5): 2193-2198

11. Khalil MS, Badawy MEI (2012) Nematicidal activity of a biopolymer chitosan at different molecular weights against root-knot nematode, Meloidogyne incognita. Plant Protect Sci 48(4): 170-178.

12. Phaniendra A, Jestadi D, Periyasamy L, (2015) Free radicals: properties, sources, targets, and their implication in various diseases. Indian J Clin Biochem 30(1): 11-26.

13. Jose AO Brien, Arsalan Daudi, Vernon S Butt, Paul Bolwell G (2012) Reactive oxygen species and their role in plant defence and cell wall metabolism. Planta 236(3): 765-779. 